\title{
Efectos de los corticoides inhalatorios sobre el crecimiento en niños con asma
}

Use of inhaled corticosteroids in children with asthma and effects on growth

Pruteanu A et al. The Cochrane Collaboration. Publicado en The Cochrane Library 2014, Issue 7.

\section{Objetivos}

Evaluar si el incremento en la dosis de corticoides inhalatorios se asocia con reducción de la velocidad de crecimiento, ganancia de peso, y/o a cambios en la maduración esquelética de los niños con asma.

\section{Fuentes de datos}

Cochrane Central of Controlled Trials (CENTRAL), MEDLINE, EMBASE, CINAHL, AMED, PsycINFO y búsquedas manuales en revistas de medicina respiratoria y en resúmenes de congresos.

\section{Selección de estudios}

Ensayos clínicos controlados aleatorizados con grupos paralelos que hubieran incluido niños de 1 a 17 años con diagnóstico de asma persistente. Cada grupo de tratamiento debía haber usado el mismo corticoide inhalatorio $(\mathrm{Cl})$ en dos o más diferentes dosis, pero con la misma vía de administración y por un período de al menos tres meses. Fue definido grupo intervención al representado por quienes habían sido asignados a $\mathrm{Cl}$ en bajas dosis y grupo control, al representado por las ramas de altas dosis.

\section{Extracción de datos}

Los datos fueron extraídos por dos revisores independientes. Los estudios seleccionados debían contener información sobre las características de la población incluida, el tipo de $\mathrm{Cl}$ utilizado, la dosis y la frecuencia de su administración, el dispositivo inhalatorio, la duración y la adherencia al tratamiento en ambos grupos, la presencia de cointervenciones, el número de participantes seleccionados y que completaron el protocolo, así como el número y las razones de la pérdida de participantes durante el seguimiento.

\section{Resultados principales}

El resultado primario fue la velocidad de crecimiento (expresada en $\mathrm{cm} / \mathrm{año}$ ), obtenida por la medición de la altura en distintos puntos de tiempo durante el estudio. Los resultados secundarios fueron los cambios observados en la desviación estándar de la velocidad de crecimiento (SD), en la talla (cm), en el peso ( $\mathrm{kg}$ o puntaje $\mathrm{z}$ ), en el índice de masa corporal (IMC), y en la maduración esquelética.

Tabla 1. Resultados principales del tratamiento en altas dosis de corticoides inhalatorios en la reducción de la velocidad de crecimiento, ganancia de peso y cambios en la maduración esquelética en niños con asma persistente.

\begin{tabular}{|c|c|c|c|c|c|}
\hline \multirow[b]{2}{*}{ Resultado evaluado } & \multirow{2}{*}{$\begin{array}{l}\text { Tiempo a la } \\
\text { evaluación }\end{array}$} & \multicolumn{2}{|c|}{ Media por grupo (IC95\%) } & \multirow{2}{*}{$\begin{array}{l}\text { Diferencia de medias } \\
\text { (IC955) }\end{array}$} & \multirow{2}{*}{$\begin{array}{l}\text { Participantes } \\
\text { (estudios) }\end{array}$} \\
\hline & & Bajas dosis & Altas dosis & & \\
\hline \multirow[t]{2}{*}{ Cambios en la talla } & 3 meses & $1,19 \mathrm{~cm}$ & $1.34 \mathrm{~cm}$ & $-0,15 \mathrm{~cm}(-0,28 \mathrm{a}-0,02)$ & $944(9)$ \\
\hline & 12 meses & $4.81 \mathrm{~cm}$ & $4,56 \mathrm{~cm}$ & $0,25 \mathrm{~cm}(-0,04 \mathrm{a} 0,54)$ & $548(4)$ \\
\hline Cambios en el índice de masa corporal & \multirow[t]{3}{*}{12 meses } & $0,5 \mathrm{~kg} / \mathrm{m}^{2}$ & $0,7 \mathrm{~kg} / \mathrm{m}^{2}$ & $-0,20 \mathrm{~kg} / \mathrm{m}^{2}(-0,49$ a 0,09$)$ & 408 (1) \\
\hline Cambio en la maduración esquelética & & 1,13 años & 0,95 años & $0,18(0,02$ a 0,34$)$ & $181(1)$ \\
\hline Velocidad de crecimiento & & $5,94 \mathrm{~cm} /$ año & $5,74 \mathrm{~cm} /$ año & $0,20 \mathrm{~cm} / \mathrm{año} 0(0,02$ a 0,39$)$ & $728(4)$ \\
\hline
\end{tabular}

\section{Conclusiones}

En niños prepúberes con asma leve a moderada persistente se observó una diferencia pequeña pero estadísticamente significativa en la velocidad de crecimiento, a favor del uso de dosis bajas de $\mathrm{Cl}$.

Fuentes de financiamiento/conflicto de interés de los autores: No referida

\section{Comentario}

Los corticoides inhalatorios $(\mathrm{Cl})$ constituyen el tratamiento de primera línea para el asma persistente, tanto en niños como en adultos. Distintos ensayos clínicos ${ }^{1,2,3}$ han demostrado su eficacia para el control de los síntomas, para la disminución de las exacerbaciones y las hospitalizaciones, para la mejoría de la función pulmonar y la calidad de vida y para la disminución de la mortalidad.

Sin embargo, los posibles efectos adversos sistémicos relacionados con el uso de $\mathrm{Cl}$ a largo plazo, principalmente sobre el crecimiento, sigue siendo un tema controvertido y motivo de preocupación de los padres y los médicos.

Si bien en el largo plazo, la velocidad de crecimiento fue mayor en el grupo que recibió bajas dosis de $\mathrm{Cl}$, es interesante destacar que cuando los niños fueron evaluados tempranamente (a los tres meses), las diferencias de cambio en la talla fueron favorable a los tratados con altas dosis de $\mathrm{Cl}$, lo que podría estar vinculado con un más rápido control del asma en este subgrupo.

\section{Conclusiones del comentador}

Se necesitan estudios mejor diseñados y con mayor tiempo de seguimiento para poder evaluar la repercusión de la dosis de $\mathrm{Cl}$ sobre la velocidad de crecimiento en niños con asma.

David Cólica [ Servicio de Medicina Familiar y Comunitaria del Hospital Italiano de Buenos Aires. david.colica@hospitalitaliano.org.ar ]

Colica D. Efectos de los corticoides inhalatorios sobre el crecimiento en niños con asma. Evid Act Pract Ambul. 2016;19(3):87. Comentado de: Pruteanu A y col. Inhaled corticosteroids in children with persistent asthma: dose-response effects on growth. Cochrane Database Syst Rev. 2014:CD009878. PMID: 25030199.

Referencias:

1. Sharek Py col. Beclomethasone for asthma in children: effects on linear growth. Cochrane Database of Systematic Reviews 2000, Issue 2. [DOI:10.1002/14651858.CD001282]

2. Pedersen $S$ y col. Efficacy and safety of three ciclesonide doses vs placebo in children with asthma: the rainbow study. Respiratory Medicine 2010;104 (11):1618.

3. Lipworth B. Systemic Adverse Effects of Inhaled Corticosteroid Therapy. A Systematic Review and Meta-analysis. Arch. Interm. Med. 1999;159:941-55. Articulo comentado en Evidencia Actualización en la Práctica Ambulatoria - Vol. 3 Número 1: Enero-Febrero 2000. 